\title{
Heart valve surgery in the United Kingdom: present practice and future trends
}

The surgery of heart valve disease raises many questions. Should we conserve or replace the diseased valve? What should the replacement valve be-a homograft, a bioprosthetic valve, or a mechanical valve? Isn't valve disease becoming less common anyway?

Test reports, clinical comparisons, and longitudinal follow up studies, particularly those focusing on the very latest artificial heart valve are reminiscent of car tests in motoring magazines-with the latest model, incorporating state of the art technology, giving superior performance (under manufacturer's test conditions) . . . and costing just that bit more. This plethora of scientific advice about which valve to choose can be confusing and individual reports are not necessarily widely representative or capable of extrapolation.

Comprehensive information on the pattern of operations for heart valve replacement has been available on a national basis in the United Kingdom since 1986, when the United Kingdom Heart Valve Registry was established. This registry, funded by the Department of Health, receives data from all National Health Service cardiac surgical units in the United Kingdom and provides unique information on the changes in patient numbers, on demographics data, and on the preferred types of artificial valves currently being implanted by cardiac surgeons in the United Kingdom.

Although the national registry cannot yet be expected to answer the vexed question, "what artificial heart valve should we use?", it already provides answers to other questions.

What number of heart valve replacement operations are carried out in the United Kingdom? About 5000 artificial valves are implanted each year in the United Kingdom, in just over 4500 patients. These totals remained remarkably stable in the period 1986-89 (table 1). They are similar to those reported in the United Kingdom Cardiac Surgical Register-4791-4873 heart valve opera-

Table 1 Number of patients and heart valve implants in United Kingdom 1986-89

\begin{tabular}{lll}
\hline Year & Patients & Valves \\
\hline 1986 & 4665 & 5217 \\
1987 & 4525 & 5087 \\
1988 & 4706 & 5204 \\
1989 & 4569 & 5058 \\
\hline
\end{tabular}

Table 2 Heart valve implants by type and position in 1986-89

\begin{tabular}{lllrlll}
\hline Year & Single & Double & Triple & Aortic & Mitral & Tricuspid \\
\hline 1986 & 4216 & 490 & 7 & 2817 & 2353 & 47 \\
1987 & 4104 & 475 & 11 & 2779 & 2258 & 50 \\
1988 & 4216 & 482 & 8 & 2859 & 2287 & 58 \\
1989 & 4101 & 458 & 12 & 2934 & 2086 & 38 \\
\hline
\end{tabular}

tions per year in the years $1977-1980$ (J Cleland, personal communication). This remarkable consistency in numbers of valve operations in the United Kingdom contrasts with the considerable increase in coronary graft operations over the same time period. In the late 1970s fewer than 4000 coronary graft operations were carried out each year. By 1985 the annual figure had risen to over 12000 operations.

Single valve replacement procedures account for around $90 \%$ of all valve operations. Ten per cent are double valve replacements, and triple valve replacement operations are rare, accounting for less than $0.2 \%$ of all valve procedures. As with total numbers of implants, these figures have shown remarkably little change over the past few years (table 2).

Aortic valve replacement is now the commonest procedure: it accounts for around $60 \%$ of procedures. Mitral valve replacements account for almost $40 \%$, and only around $1 \%$ of valve replacements are tricuspid replacements.

These data suggest that the expected overall reduction in valve surgery numbers as rheumatic valve disease became less common has not in fact happened. A reduction in rheumatic abnormalities has presumably been balanced by an increase in valve replacement procedures for degenerative valve disease. This could also explain the increasing proportion of aortic over mitral replacements over the past five years. (aortic 54\%-59\%, mitral $45 \%-40 \%$ ).

What artificial valves do we use?

This has changed considerably over the past four or five

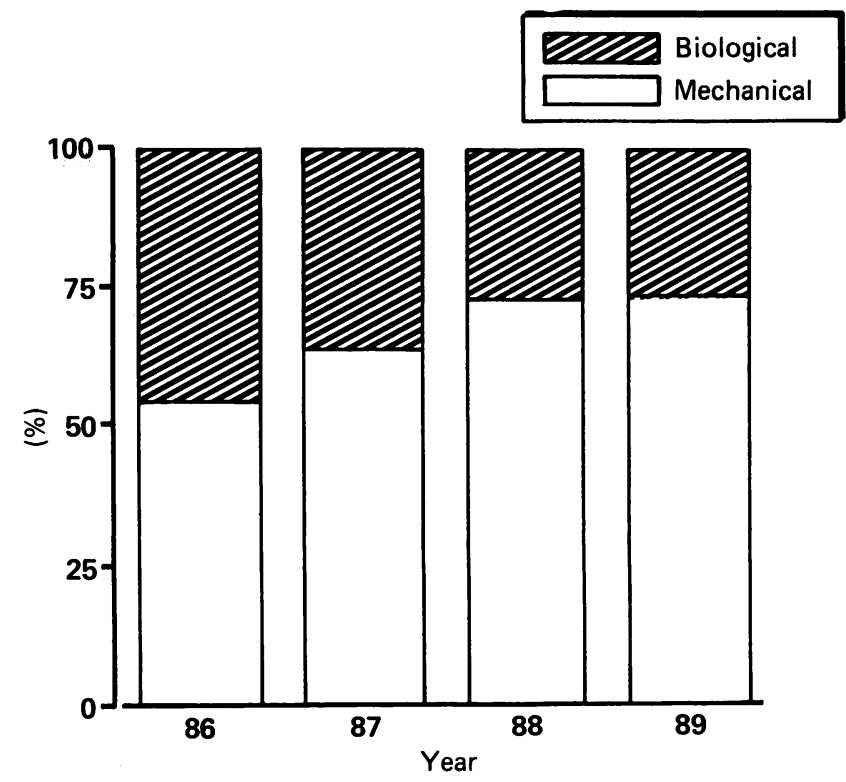

Figure 1 Percentage use of biological and mechanical heart valve prostheses in the United Kingdom (1986-89) (data from United Kingdom Heart Valve Registry). 


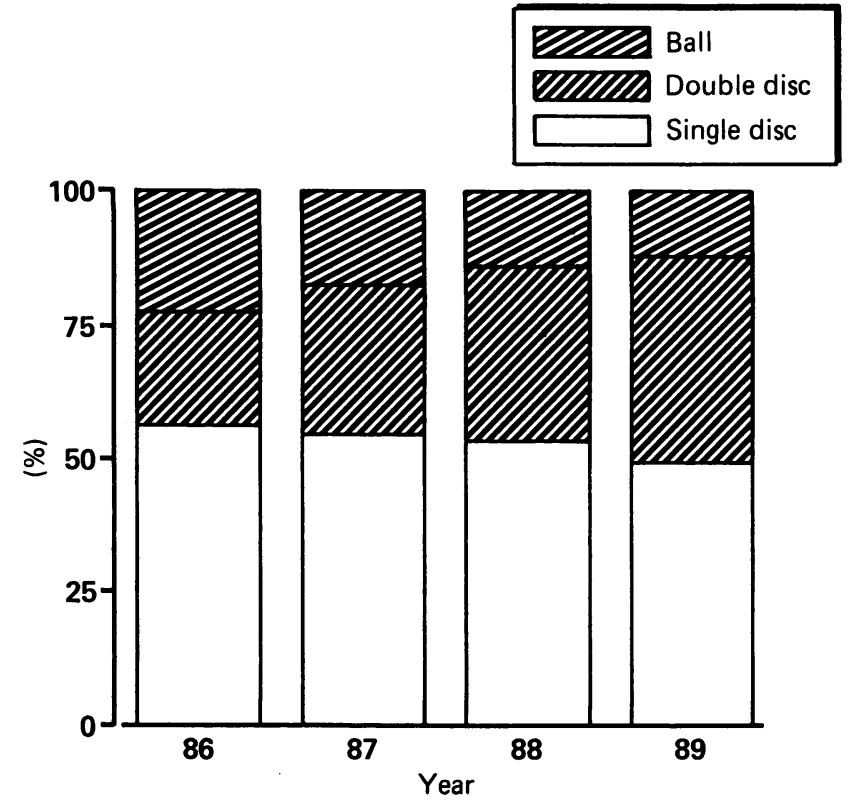

Figure 2 Percentage use of main types of mechanical heart valve prostheses in the United Kingdom (1986-89) (data from United Kingdom Heart Valve Registry).

years. In 1986 about $54 \%$ of valves implanted in the UK were mechanical valves, compared with $46 \%$ bioprosthetic valves. Homograft valves account for less than $1 \%$ of total implants. In 1987-88 an increased preference for mechanical valves resulted in a $70 \%$ to $30 \%$ split between mechanical and bioprosthetic valves. The situation seems to have reached a steady state with little further change over the past two years (fig 1 ).

There are also trends in the use of particular valve types. Over the past four years single disc valves were the most commonly used mechanical valves. The use of ball valves declined and there was a significant corresponding increase in the use of bi-leaflet valves (fig 2). For the year 1989, the figures were $48 \%$ single leaflet, $37 \%$ bi-leaflet, and $15 \%$ ball valves. In the bioprosthetic valve group, the preference for porcine over bovine pericardial valves has increased since 1986 when $75 \%$ of bioprosthetic valves implanted were porcine. By 1989 the proportion of pericardial valves had fallen to $15 \%$, presumably reflecting doubts over the long term durability of pericardial valves.

These data indicate a clear shift in surgical preference in the period 1986-89 towards mechanical valves. No single factor is obvious in determining this trend, but it seems likely that the perceived requirement for re-operation with bioprosthetic valves convinces surgeons and cardiologists more than the potential of avoiding anticoagulation in some patients. Whether this perception can be supported by hard data is quite another matter. It is interesting, however, to see such a clear trend, particularly when patients are getting older (37\% over 65 years in 1989) and when $60 \%$ of all replacements are aortic valves, more often than not in patients in sinus rhythm.

\section{What of the future?}

Whatever else happens, it seems certain that the average age of patients requiring heart valve surgery will continue to increase. It has been estimated that $40 \%$ of valve surgery patients in the 1990s will be over 65 years of age. Although valve surgery in the elderly patient may be performed with very acceptable results, operative mortality and postoperative morbidity have been shown to be increased. Careful preoperative assessment and identification of major risk factors in elderly patients should improve results further.

Conservative valve surgery, particularly in the mitral position, is likely to increase as surgeons gain more experience in the techniques pioneered by Carpentier et al in France ${ }^{1}$ and Cosgrove et al in the USA. ${ }^{2}$ Currently, conservative procedures account for around $10 \%$ of all mitral valve operations but only $0.5 \%$ of aortic procedures. Homograft aortic valve replacements make up around $2 \%$ of all aortic replacements. It seems likely, therefore, that most heart valve surgery in the United Kingdom population will continue to require the replacement of affected valves by some form of artificial valve. ${ }^{3}$ The current preference for mechanical valves may well continue at present levels, although this part of the whole equation is difficult to predict.

Long term information from the national registries should provide reliable data upon which such choices may be made in the future. At the very least, registry information will let us know the extent of the correlation, if any, between the valves we think we should use and the valves we actually do use.

Cardiothoracic Surgical Unit,

KENNETH M TAYLOR

Royal Postgraduate Medical School,

Hammersmith Hospital,

Du Cane Road, London W12 0NN

1 Carpentier A, Chauvaud S, Fabiani JN, et al. Reconstructive surgery of mitral valve incompetence. $J$ Thorac Cardiovasc Surg 1980;79:338-48. Cosgrove DM, Chavez AM, Lytle BW, et al. Results of mitral valve reconstruction. Circulation 1986;74:82-7.

3 Taylor KM. Acute failure of artificial heart valves. BMJ 1988;297:996-7. 